\section{Prevalence of dermatophytosis in dogs and cats in Egypt}

\section{Nashwa Waly 1,2 , Rehab Kamel ${ }^{1}$, Laila Ahmed ${ }^{1}$, Mady Ismail ${ }^{3}$}

1 Dept Of Animal Medicine, Faculty Of Veterinary Medicine, Assiut University, Assiut, Egypt

2 University Of Bristol, Bristol, Uk

3 Dept Of Plant Biology, Faculty Of Science, Assiut University, Assiut, Egypt

\section{OBJECTIVES}

Study the prevalence and identify different Dermatophytes affecting pets in the area.

\section{METHODS}

103 symptomatic and asymptomatic animals were recruited; 53 cats and 20 dogs were clinical cases; 30 animals were from a local pet-shop (18 cats and 12 dogs). All animals were given a full clinical and Wood's lamplight examination; their hair brushings were cultured using standard techniques.

Descriptive statistics were used to summarize the results $\&$ identify emerging patterns.

\section{RESULTS}

$69 \%$ of symptomatic and $16.7 \%$ of asymptomatic cats in the clinical group were positive for dermatophytes. 53\% asymptomatic pet-shop cats also cultured positive.

$33.3 \%$ of symptomatic and $9.1 \%$ of asymptomatic dogs from the clinical group cultured positive for dermatophytes. $100 \%$ of symptomatic \& $44.4 \%$ of asymptomatic pet-shop dogs were positive for dermatophytes.

Six different dermatophytes species were identified from clinical cat cases (Microsporum (M.) canis, Epidermophyton floccosum, M. praecox, M. gypseum, $M$. nanum and Trichophyton interdigitale). M. praecox was isolated from clinical cat group $(n=3)$.

Three species of dermatophytes were isolated from dogs in the clinical case group. M. canis predominated (60\%), followed by Trichophyton mentagrophytes and Epidermophyton floccosum. Microsporum gypseum was the predominant species $(42.86 \%)$ in pet-shop dogs.

The incidence of dermatophytosis increased in the spring $\&$ winter. Young cats were more likely to be positive for dermatophytosis $(P=0.026)$.

\section{STATEMENT}

Incidence of dermatophytosis is higher in this area compared to other countries. Strict hygienic measures should be followed in pet-shops as they have a high infection rate which can lead to infection of newly introduced cats and dogs.

\section{A description of owner-reported flea control measures and skin disease in a cohort of young cats}

\section{Sophie Tyler, Claire Roberts, Aiden Foster, Natalie Barnard, Jane Murray}

University of Bristol, Bristol, UK

\section{OBJECTIVES}

Feline skin disease is prevalent within primary care veterinary practice. This study describes owner-reported skin disease within a cohort of pet cats. The type of flea control product used (prescription/non-prescription) was assessed for association with owner-reported skin disease at age 2.5 years.

\section{METHODS}

Study data were collected from two owner-completed questionnaires (completed when cats were aged 2-4 months (T1) and 2.5 years (T2)) for cats enrolled on a long-term longitudinal study ('Bristol Cats' study). Chisquared analysis was used to test for association between owner-reports of flea control products used at T1 and at both T1 and T2 (categorised as prescription/ non-prescription products), and presence of ownerreported feline skin disease at age 2.5 years.

\section{RESULTS}

Feline skin disease at age 2.5 years was reported by owners of $55 / 1150(4.8 \%, 95 \% \mathrm{Cl} 3.7-6.2 \%)$ study cats. Most $(30 / 55,54.5 \%)$ had not presented their cat to a veterinarian for examination of the condition.

Excluding owners who did not know the brand of flea treatment used at T1 and/or T2 and those reporting using both prescription and non-prescription products, the majority $(237 / 317,74.8 \%)$ used prescription treatments at $\mathrm{T} 1$ and at T2.

There was no significant association between owner use of prescription/non-prescription feline flea control at T1 $(P=0.7)$ or at both T1 and T2 $(P=0.44)$ and ownerreported skin disease in cats at 2.5 years.

\section{STATEMENT}

Over $50 \%$ of owners who reported skin disease did not seek veterinary attention. The category of flea control product (prescription/non-prescription) used did not affect the prevalence of owner-reported skin disease. 